\title{
Technè
}

La science au service de l'histoire de l'art et de la préservation des biens culturels

46 | 2018

Science et conservation

\section{Caractérisation d'un mastic prêt à l'emploi peu connu pour le comblement superficiel des céramiques : le Flügger Filler Interior}

Description of a little-known, ready-to-use filler for superficial gaps in ceramics: Flügger Filler Interior

Patricia Dupont-Aulagnier, David Giovannacci et Léa Rocourt

\section{OpenEdition}

Journals

Édition électronique

URL : http://journals.openedition.org/techne/498

DOI : $10.4000 /$ techne.498

ISSN : 2534-5168

Éditeur

C2RMF

Édition imprimée

Date de publication : 1 décembre 2018

Pagination : 109-113

ISBN : 978-2-11-152829-1

ISSN : 1254-7867

\section{Référence électronique}

Patricia Dupont-Aulagnier, David Giovannacci et Léa Rocourt, « Caractérisation d'un mastic prêt à l'emploi peu connu pour le comblement superficiel des céramiques : le Flügger Filler Interior », Technè [En ligne], 46 | 2018, mis en ligne le 19 décembre 2019, consulté le 22 juillet 2020. URL : http:// journals.openedition.org/techne/498; DOI : https://doi.org/10.4000/techne.498

\section{(ब) $\Theta \Theta$}

La revue Technè. La science au service de l'histoire de l'art et de la préservation des biens culturels est mise à disposition selon les termes de la Licence Creative Commons Attribution - Pas d'Utilisation Commerciale - Pas de Modification 4.0 International. 
Patricia Dupont-Aulagnier

David Giovannacci

Léa Rocourt
Caractérisation d'un mastic prêt à l'emploi peu connu pour le comblement superficiel des céramiques : le Flügger Filler Interior
Description of a little-known, ready-to-use filler for superficial gaps in ceramics: Flügger Filler Interior
Résumé. Le comblement des manques superficiels (éclats, manques le long des lignes de cassure) est une problématique récurrente en restauration de céramiques. Dans la pratique, les restaurateurs utilisent certains produits prêts à l'emploi, parmi lesquels le Modostuc et le Flügger Filler Interior, car ils sont très faiblement toxiques et d'une mise-en-cuvre aisée. La perception des utilisateurs, lors de l'emploi de ces deux produits, laisse présumer certaines différences, notamment au niveau de leur rugosité, de leur dureté et de leur adhérence à la pâte céramique. L'objet de cette étude est de mettre en place une procédure expérimentale simple et adaptée permettant de différencier, par la mesure, les propriétés mécaniques de ces produits. Les résultats obtenus indiquent clairement des dissimilitudes et pourront servir de base dans le choix du produit le plus adapté à chaque céramique traitée.

Mots-clés. Procédure expérimentale, Flügger Filler Interior, Modostuc, mesure, rugosité, dureté, adhérence.

\begin{abstract}
Filling in superficial gaps (chips, losses along cracks) is a recurrent problem in ceramic restoration. In practice, restorers employ certain ready-to-use products, among which are Modostuc and Flügger Filler Interior, because they have low toxicity and are easy to use. While employing these two products, users noticed differences, particularly in their roughness, hardness and adhesion to the ceramic. The purpose of this study was to establish a simple, specially adapted, experimental procedure enabling us to differentiate, by measuring, the mechanical properties of these products. The results obtained clearly indicated the differences and may now serve as a basis for choosing the most suitable product to use on each ceramic in need of restoration.
\end{abstract}

Keywords. Experimental procedure, Flügger Filler Interior, Modostuc, amount, roughness, hardness, adhesion.

\section{Introduction}

Les céramiques sont très vulnérables aux chocs mécaniques. Les éclats ou pertes de matières le long des lignes de cassure sont donc des altérations très fréquentes. Ces manques superficiels, de dimensions restreintes, peuvent être comblés à l'aide de mastic prêt à l'emploi. Le mastic le plus couramment utilisé dans le domaine de la conservation-restauration des céramiques est le Modostuc, mastic de base polyvinylique. Un autre produit, peu connu en France, mais très employé au Royaume-Uni et aux États-Unis, est le Flügger Filler Interior, mastic de base acrylique. Les utilisateurs ont noté des différences de propriétés entre ces deux produits : le Flügger ${ }^{1}$ serait plus lisse, plus dur et plus adhérent à la céramique que le Modostuc. Afin de confirmer ou non, par des mesures scientifiques, ces observations empiriques, il a paru utile de consacrer une étude 2 à cette question.

Le Flügger, de couleur blanche (seule formulation disponible à la vente), est testé en regard du Modostuc blanc qui sert uniquement de référence. Deux formulations colorées à base de Flügger, obtenues, respectivement, par ajout de pigment et de peinture acrylique, enrichissent l'étude, afin d'évaluer leur influence sur les propriétés testées (la rugosité, la dureté et l'adhérence).

\section{Procédure expérimentale}

La procédure expérimentale mise en place doit permettre de différencier les produits testés au travers de tests qualitatifs et semi-quantitatifs.

\section{Rugosité}

La rugosité est une caractéristique de l'état de surface d'un matériau solide et peut se définir comme un écart de hauteur d'une surface par rapport à sa forme générale. Sa mesure sur des mastics secs et non poncés permet donc de comparer leur état de surface à l'aide de valeurs quantifiées. Parmi la 
centaine de paramètres permettant de caractériser l'état d'une surface, « $S a$ » est l'indicateur de rugosité le plus utilisé et correspond à l'écart moyen arithmétique. Il donne une information globale sur l'ensemble de la surface mesurée.

Le rugosimètre Phaseview permet l'accès à $S a$ avec, pour avantages, la rapidité de la mesure (quelques secondes) et le choix du mode d'acquisition le plus adapté à la forme générale de l'éprouvette. Pour des éprouvettes non planes, la mesure repose sur le déplacement d'une caméra entre deux positions extrêmes choisies ${ }^{3}$, entre lesquelles trente images seront prises à intervalle régulier. Pour chaque image, un algorithme permet de ne conserver que les points nets et ainsi de reconstruire la topographie de la surface étudiée sur ces points. Trois mesures sont effectuées par éprouvette dans trois zones différentes afin d'obtenir la moyenne et l'écarttype pour chaque produit testé.

\section{Dureté}

La dureté est définie comme la capacité d'un matériau à résister à la pénétration. De nombreux essais permettent de la mesurer selon la nature du matériau. Ils ont tous un principe commun : la mesure de l'empreinte laissée par un indenteur appliqué sur le matériau avec une force connue. Plus l'empreinte est large ou profonde, plus le matériau est mou.

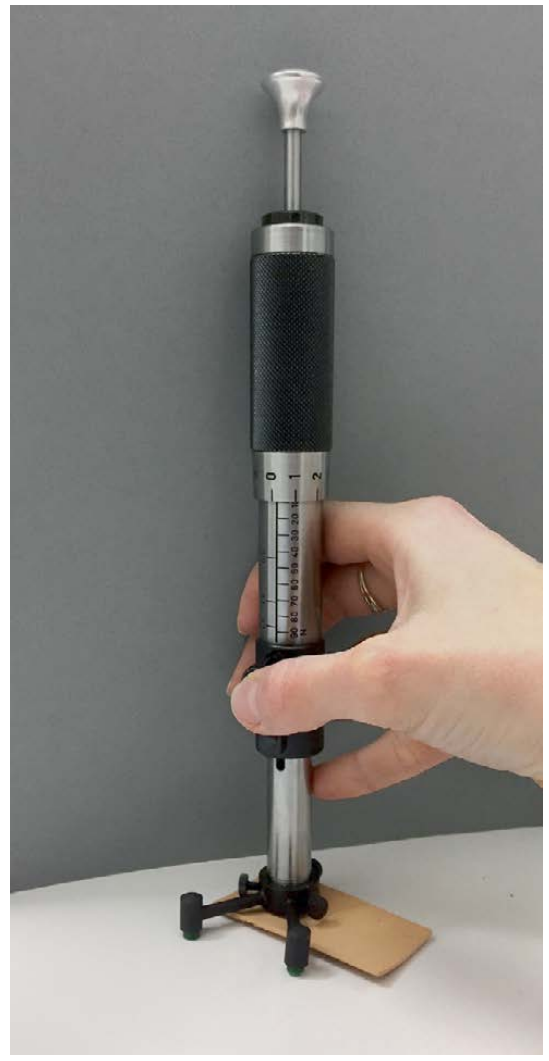

Fig. 1. Impactomètre à bille et éprouvette. (c) L. Rocourt.
L'impactomètre à bille (modèle 305 de type Wegner, Erichsen) est un appareil transportable et facile d'utilisation. Une force de 10 Newton $^{4}$ est appliquée perpendiculairement à la surface de l'éprouvette (fig. 1). Les mesures des diamètres des empreintes sont réalisées à l'aide du rugosimètre (incertitude de mesure de l'ordre du 1/10 $\mu \mathrm{m}$ ). Cinq mesures sont effectuées par matériau testé.

\section{Adhérence}

L'adhérence est la force nécessaire pour séparer deux matériaux réunis par une surface commune. Cette mesure s'effectue grâce à un extractomètre de terrain (WIGAmeter FS et DYNNA Z16Fs), habituellement utilisé pour tester l'efficacité d'une réparation sur les murs (béton, pierre, etc). La traction sera appliquée à une pastille collée à la surface de l'éprouvette (fig. 2). Le dispositif expérimental a été adapté aux éprouvettes de l'étude, avec l'utilisation d'une plaque en aluminium de grande dimension percée d'un disque de $50 \mathrm{~mm}$ de diamètre et intercalée entre l'éprouvette et l'extractomètre. La traction est appliquée en tournant une manivelle qui permet à la tige filetée de remonter. La fin de l'essai est définie lorsque la pastille est libérée de l'éprouvette. La valeur mesurée de la force est alors nulle. Trois mesures sont réalisées par formulation, soit douze éprouvettes au total. En plus des mesures, le comportement mécanique des éprouvettes lors du test (c'est-à-dire la façon dont la pastille se libère du reste de l'éprouvette) rend compte de la cohésion de la céramique, de l'adhésion entre le mastic et la céramique et de la cohésion du mastic lui-même.

\section{Présentation des matériaux et préparation des éprouvettes}

Les deux matériaux étudiés sont des dispersions de résine dans l'eau. La charge est essentiellement du carbonate de calcium ${ }^{5}$. Le Modostuc et le Flügger présentent l'avantage d'être facilement mis en œuvre, grâce à leur consistance ajustée pour le masticage, mais surtout d'avoir une toxicité très faible ${ }^{6}$. Leur séchage se fait par évaporation de l'eau et/ou son absorption dans le substrat, ce qui entraîne un retrait.

Les conditionnements sont ouverts simultanément, les mastics sont utilisés purs et sans dilution, pour garantir leur fraîcheur et optimiser la reproductibilité des mesures.

Les matières colorantes, la peinture acrylique et les pigments ont été introduits à $9 \%$ (en masse) dans le Flügger. La peinture acrylique Liquitex ${ }^{\circledR}$, extra-fine heavy body, de couleur terre de Sienne naturelle, est retenue : d'une part, cette marque fait référence et est couramment utilisée dans le domaine de la conservation-restauration ; d'autre part, une « terre " permet d'avoir la même couleur en peinture et en pigments. Le pigment utilisé est la terre de Sienne naturelle de marque Kremer, avec une granulométrie inférieure à $63 \mu \mathrm{m}$. Il n'est pas re-broyé avant mélange. 


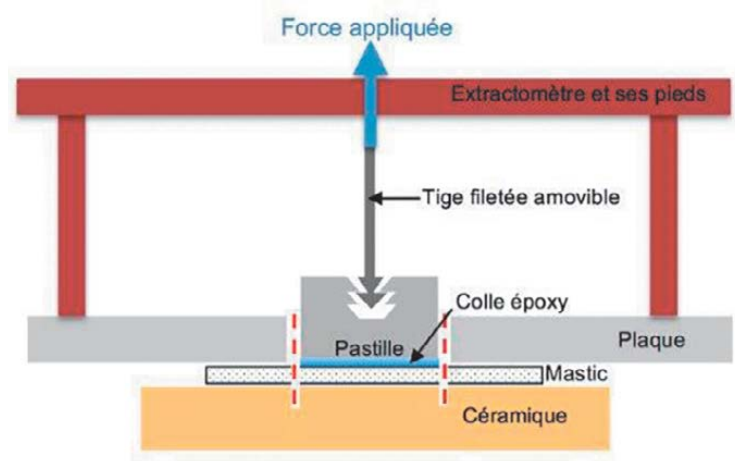

Fig. 2. Schéma montrant le principe du test d'adhérence, vue en coupe. (C) L. Rocourt.

Les matières colorantes sont incorporées et mélangées à l'aide d'une spatule en métal, le plus rapidement possible (afin d'éviter que le mélange ne sèche avant l'application), jusqu'à l'obtention d'une coloration homogène.

Le test de rugosité requiert des éprouvettes dont la surface est suffisamment grande pour effectuer plusieurs mesures (le champ de mesure maximal étant $2 \times 2,5 \mathrm{~mm}^{2}$ ), mais dont l'épaisseur est faible afin d'éviter de trop grandes déformations ou fissurations lors du séchage. Les éprouvettes ont été réalisées dans des moules en silicone dont les dimensions sont les suivantes : 64 × 34,5 x 2 mm. Les éprouvettes sont laissées une semaine dans une pièce dont le climat est contrôlé à une humidité relative de $50 \%$ à $20{ }^{\circ} \mathrm{C}$. Après séchage, l'observation des éprouvettes à l'œil nu a permis de retenir les plus homogènes d'entre elles, par leur forme générale (la plus plane possible) et par leur surface (celle qui a séché à l'air libre, la plus lisse possible). Elles ont été manipulées uniquement par les bords et ne sont pas poncées. Le même type d'éprouvette est choisi pour l'essai de dureté.

Le test d'adhérence nécessite un support en céramique non glaçurée et poreuse. Des carreaux de biscuit en faïence rouge fabriqués industriellement ont été recouverts d'une couche de mastic de $2 \mathrm{~mm}$ d'épaisseur ${ }^{7}$, à l'aide d'une spatule droite. Une pastille taraudée en aluminium a été collée en leur centre (à l'aide de colle époxy Sader ${ }^{\circledR}$ progressive $2 \mathrm{~h}$ ), puis un sillon a été créé tout autour à l'aide d'un disque diamanté monté sur micro-tour (fig. 2). Il permet de limiter la sollicitation mécanique à la zone collée.

\section{Résultats et discussion}

\section{Rugosité}

Les résultats de rugosimétrie révèlent que le Flügger blanc est plus lisse, que le Modostuc, avec en moyenne, $\mathrm{Sa}_{\mathrm{Fblanc}}=5,1 \pm$ $1,6 \mu \mathrm{m}$ et $\mathrm{Sa}_{\text {Modostuc }}=7,8 \pm 1,1 \mu \mathrm{m}$. Le logiciel couplé au rugosimètre permet une reconstruction 3D des surfaces observées
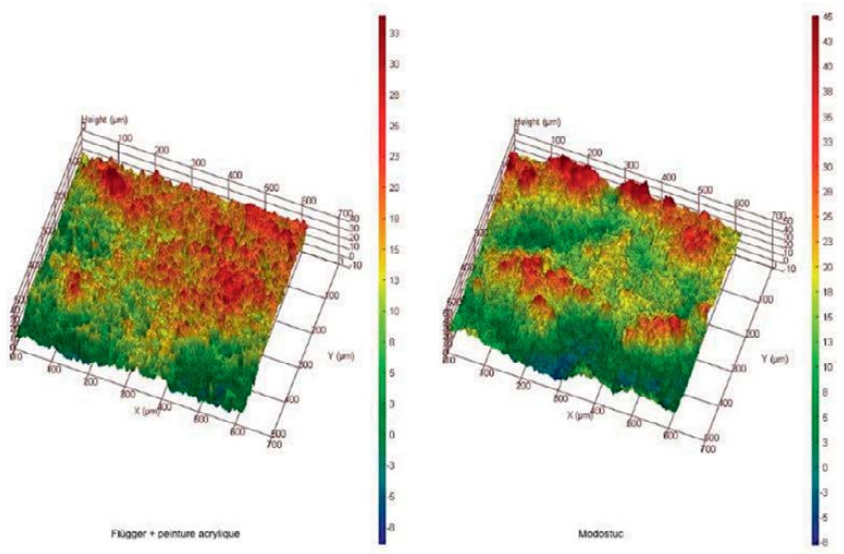

Fig. 3. Vues 3D de la surface du Flügger blanc et du Modostuc. (c) L. Rocourt.

et met en évidence les différences entre le Flügger blanc et le Modostuc (fig. 3).

De plus, l'ajout de matière colorante influence directement la rugosité : l'éprouvette Flügger additionnée de peinture acrylique est la plus lisse des quatre formulations, avec $\mathrm{Sa}_{\mathrm{F}+\text { peint. }}=4,2 \pm 1,2 \mu \mathrm{m}$. Les additifs et le liant présents dans la peinture ont certainement permis au mastic de mieux « napper » la surface. Au contraire, l'ajout de pigments rend la surface plus rugueuse (en asséchant le mélange, certains grains de pigment s'incorporent mal et créent des défauts macroscopiques, fig. 4) avec une moyenne de $\mathrm{Sa}_{\mathrm{F}+\text { pig. }}=9,3 \pm$ $3,3 \mu \mathrm{m}$, soit plus du double de l'éprouvette Flügger additionnée de peinture acrylique.

L'hypothèse liée à la perception des utilisateurs est donc vérifiée : brut d'application, le Flügger est plus lisse que le Modostuc.

\section{Dureté}

La moyenne des cinq diamètres d'impacts mesurés, $d$, sur le Modostuc est la plus importante, soit $\mathrm{d}_{\text {Modostuc }}=1181,9 \pm 167,1$ $\mu \mathrm{m}$. C'est donc le matériau dans lequel la bille a le plus pénétré, c'est-à-dire le plus mou. Le Flügger blanc est la formulation la plus dure avec des diamètres en moyenne égaux à $\mathrm{d}_{\text {Fblanc }}=1021,2 \pm 66,7 \mu \mathrm{m}$.

Enfin, l'ajout de pigments et de peinture acrylique rend le Flügger plus mou avec des diamètres moyens égaux respectivement, à $\mathrm{d}_{\mathrm{F}+\text { pig. }}=1174,8 \pm 132,2 \mu \mathrm{m}$ et à $\mathrm{d}_{\mathrm{F}+\text { peint. }}=1069,8 \pm$ $70,1 \mu \mathrm{m}$.

\section{Adhérence}

La charge, $C$, nécessaire pour séparer le Modostuc du support en céramique est la plus faible des quatre formulations, avec $\mathrm{C}_{\text {Modostuc }}=3,2 \pm 0,3 \mathrm{~kg} / \mathrm{cm}^{2}$. Les éprouvettes de Flügger blanc ont une charge maximale à la rupture égale à $\mathrm{C}_{\mathrm{Fblanc}}=5,5 \pm$ 
$0,3 \mathrm{~kg} / \mathrm{cm}^{2}$. Le Flügger a donc une adhérence supérieure au Modostuc, la perception est vérifiée encore ici.

Les éprouvettes de Flügger teinté aux pigments ou teinté à la peinture acrylique ont des charges maximales à la rupture égales respectivement à $\mathrm{C}_{\mathrm{F}+\text { pig. }}=6,3 \pm 0,8 \mathrm{~kg} / \mathrm{cm}^{2}$ et $\mathrm{C}_{\mathrm{F}+\text { peint. }}=$ $6,6 \pm 1 \mathrm{~kg} / \mathrm{cm}^{2}$. L'ajout de pigment et de peinture acrylique augmente donc la charge maximale à la rupture de l'éprouvette.

Outre les mesures, la différence de comportement est notable entre les éprouvettes à base de Modostuc et celles à base de Flügger (fig. 5). Lors de la libération de la pastille, le Modostuc se détache à l'interface : il s'agit d'une rupture adhésive à l'interface. En revanche, lors des essais, des fissures se forment dans toute l'épaisseur des éprouvettes à base de 112 Flügger. Il y a rupture cohésive, dans la céramique. L'adhésion entre le Flügger et la pâte céramique est donc supérieure à la cohésion même de la céramique.

\section{Discussion}

D'après les résultats obtenus et résumés dans le tableau (fig. 6), le Flügger blanc est plus lisse que le Modostuc blanc. Lajout de peinture acrylique modifie les propriétés du mastic, particulièrement au niveau de son état de surface en le rendant plus lisse, tandis que l'ajout de pigment le rend moins lisse. Le Flügger est également plus dur et plus adhérent à la pâte céramique. La forte adhérence d'un mastic sur une céramique est-elle toujours un avantage ? Si cette adhérence entraîne une rupture cohésive dans la céramique en cas de choc mécanique ou de sollicitations, alors la réponse est non. Il est fondamental que ce soit uniquement le comblement qui se détache, sans endommager la céramique, comme c'est le cas pour les éprouvettes à base de Modostuc. La rupture adhésive à l'interface est souhaitable pour les produits utilisés en conservation-restauration.

La perception des utilisateurs est confirmée grâce aux tests qualitatifs (adhérence) et semi-quantitatifs (rugosité et dureté).

Bien que les résultats soient homogènes pour chaque matériau testé, une augmentation du nombre d'éprouvettes pourrait permettre d'assurer une analyse statistique plus importante.

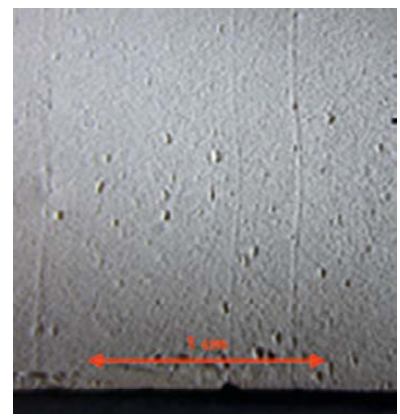

Fig. 4. Photographie en lumière rasante de la surface de l'éprouvette à base de Flügger teinté au pigment. ( ) L. Rocourt.

\section{Conclusion}

Le test le plus discriminant de cette étude est le test d'adhérence. Le Modostuc apparaît donc plutôt indiqué pour les comblements de céramiques dont la pâte est fragile ou friable et efficient dans des zones peu sollicitées. Quant au Flügger, il est à favoriser sur des céramiques dont la pâte est bien cuite avec une bonne cohésion. Étant plus résistant grâce à sa bonne adhérence, il sera plus adapté pour les comblements dans des zones très sollicitées telles que les bords, mais aussi pour les éclats de faible épaisseur (pour lesquels le Modostuc a tendance à se détacher lors du ponçage).

La dureté d'un comblement peut être un avantage pour éviter les enfoncements lors des manipulations ou des chocs. Les céramiques architecturales (carreaux, bas-reliefs, etc.) subissent souvent des sollicitations mécaniques sur leurs bords ou leurs parties saillantes lors du remontage. Si la pâte de la céramique a une bonne cohésion (la compatibilité et l'esthétique devant également être prises en compte), l'emploi du Flügger peut être un choix adapté et judicieux.

Enfin, la méthodologie appliquée reste simple et facilement reproductible, voire adaptable en atelier. La force de traction de l'extractomètre de terrain pourrait par exemple être reproduite à l'aide d'un câble et d'une poulie reliée à des poids. Les procédures expérimentales mises au point pourront très bien servir pour d'autres essais sur des supports en céramique différents (à pâte vitrifiée), ou avec des formulations incluant d'autres charges par exemple.

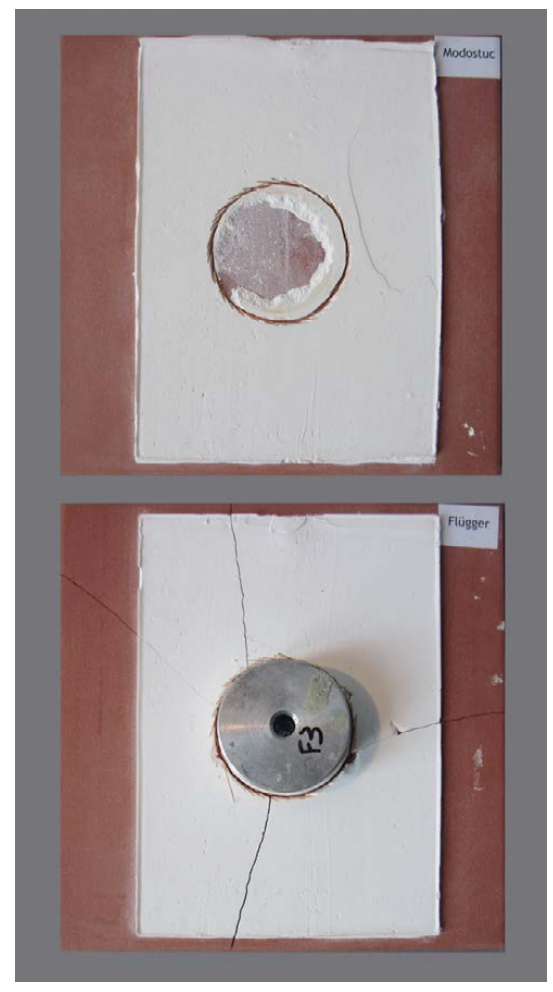

Fig. 5. Éprouvettes de Modostuc et de Flügger après le test d'adhérence. $\odot$ L. Rocourt. 


\begin{tabular}{|c|c|c|c|c|}
\hline & Modostuc & Flügger & Flügger + peinture acr. & Flügger + pigments \\
\hline \multirow{2}{*}{$\begin{array}{l}\text { Rugosité } \\
\text { (Sa, } \mu \mathrm{m}, \text { moy. et } \\
\text { écart-type) }\end{array}$} & $\begin{array}{l}7,760 \\
(1,102)\end{array}$ & $\begin{array}{l}5,120 \\
(1,627)\end{array}$ & $\begin{array}{l}4,207 \\
(1,236)\end{array}$ & $\begin{array}{l}9,310 \\
(3,264)\end{array}$ \\
\hline & Lisse & Très lisse & Le plus lisse & $\begin{array}{l}\text { Légèrement } \\
\text { rugueux }\end{array}$ \\
\hline \multirow{2}{*}{$\begin{array}{l}\text { Dureté } \\
\text { (diamètre, } \mu \mathrm{m}, \text { moy. } \\
\text { et écart-type) }\end{array}$} & $\begin{array}{l}1181,930 \\
(167,075)\end{array}$ & $\begin{array}{l}1021,247 \\
(66,664)\end{array}$ & $\begin{array}{l}1069,768 \\
(70,132)\end{array}$ & $\begin{array}{l}1174,792 \\
(132,247)\end{array}$ \\
\hline & Le plus mou & Le plus dur & $\begin{array}{l}\text { La peinture acrylique } \\
\text { diminue légèrement } \\
\text { la dureté }\end{array}$ & $\begin{array}{l}\text { Les pigments } \\
\text { diminuent la dureté }\end{array}$ \\
\hline \multirow{3}{*}{$\begin{array}{l}\text { Adhérence } \\
\text { (charge, } \mathrm{kg} / \mathrm{cm}^{2} \text {, } \\
\text { moy. et écart-type) }\end{array}$} & $\begin{array}{l}3,17 \\
(0,29)\end{array}$ & $\begin{array}{l}5,53 \\
(0,29)\end{array}$ & $\begin{array}{l}6,57 \\
(1,01)\end{array}$ & $\begin{array}{l}6,27 \\
(0,83)\end{array}$ \\
\hline & $\begin{array}{l}\text { Séparation } \\
\text { à l'interface } \\
\text { mastic-céramique }\end{array}$ & $\begin{array}{l}\text { Bris de l'éprouvette } \\
\text { dans toute } \\
\text { son épaisseur }\end{array}$ & $\begin{array}{l}\text { Bris de l'éprouvette } \\
\text { dans toute } \\
\text { son épaisseur }\end{array}$ & $\begin{array}{l}\text { Bris de l'éprouvette } \\
\text { dans toute } \\
\text { son épaisseur }\end{array}$ \\
\hline & - & - & $\begin{array}{l}\text { L'ajout de peinture } \\
\text { acrylique augmente } \\
\text { la charge maximale } \\
\text { à la rupture }\end{array}$ & $\begin{array}{l}\text { L'ajout de pigment } \\
\text { augmente la charge } \\
\text { maximale à la rupture }\end{array}$ \\
\hline
\end{tabular}

Fig. 6. Tableau récapitulatif des résultats, • rouge (résultats mauvais pour une céramique de type terre cuite ou faïence glaçurée), - orange (résultats intermédiaires), - vert (meilleurs résultats pour ce test). ( L. Rocourt.

\section{Notes}

1. Dans cet article, «Flügger» équivaut à « Flügger Filler Interior».

2. Cette étude est issue du mémoire Inp, département des restaurateurs, de Léa Rocourt encadrée par Patricia DupontAulagnier, Juliette Pollet et David Giovannacci : «Une jungle à l’Élysée ». Étude et conservation-restauration d'un médaillon de cheminée réalisé pour une salle à manger de l'Élysée, 1947-1948 (Paris, Centre national des arts plastiques) - Étude comparative de deux mastics prêts à l'emploi, utilisés pour

les comblements superficiels : le Modostuc et $l e$ Flügger.

3. Ces deux altitudes, définies par l'opérateur, correspondent au flou juste au-dessus du point le plus haut et au flou juste en dessous du point le plus bas.

4. Les essais préalables sur des éprouvettes de même type que celles qui serviront pour le test ont permis de déterminer que 10 Newton serait la force adaptée aux diverses formulations testées. En effet, au-delà de 10 Newton, l'impact induit des fissures dans l'éprouvette de Modostuc.
5. D’après Moffat, 1993 et Koob, 2000. Les concentrations et la granulométrie du carbonate de calcium ne sont pas communiquées par les fabricants.

6. Certains plastifiants et biocides contenus dans les mastics peuvent être allergisants, mais le solvant, lui, n'est pas toxique.

7. Un châblon, moule sans fond utilisé en pâtisserie, de $2 \mathrm{~mm}$ de profondeur, posé sur le support, sert de " cadre " pour appliquer le mastic.

\section{Bibliographie}

Giovannacci D. et al., 2013, "Algal colonization kinetics on roofing and façade tiles: Influence of physical parameters", Construction and Building Materials, Volume 48, November 2013, p. 670-676.

Koob S., 2000, "New Materials and Research, Flügger Acryl”, American Institute for Conservation News, vol. 25, p. 14.

Maradudin A. (ed.), 2007, Light scattering and nanoscale surface roughness, Springer, New York.

\section{Documents techniques}

Description technique de l'extractomètre de terrain, consulté le 20 janvier 2017. URL : http://www.garellodegiosa.it/ categoria_produzione_qc/proceq/ pdf/1-Concrete/DYNA_ ExtractionTester_E.pdf

Description technique de l'impactomètre, consulté le 2 février 2017. URL : https://www.erichsen.de/ surfacetesting/impact-resistance/ impact-tester-acc-to-wegner-model-305 A.

Moffat E., 1993, « Rapport analytique de l'Institut canadien de conservation (ICC), SRA 3255, Analyse Modostuc ", 17 décembre 1993 (disponible sur demande). https://www.canada.ca/fr/ institut-conservation.html 